SLAC-PUB-8232

August 1999

\title{
Dynamic Aperture Studies for SPEAR 3
}

\author{
J. Corbett, Y. Nosochkov, J. Safranek \\ Stanford Linear Accelerator Center, Stanford University, Stanford, CA 94309 \\ A. Garren \\ UCLA Center for Advanced Accelerators, Los Angeles, CA 90095
}

\begin{abstract}
The SSRL is investigating an accelerator upgrade project to replace the present $130 \mathrm{~nm} \cdot \mathrm{rad}$ FODO lattice with an $18 \mathrm{~nm} \cdot \mathrm{rad}$ double bend achromat lattice: SPEAR 3. In this paper, we review the methods used to maximize the SPEAR 3 dynamic aperture including optimization of linear optics, betatron tune, chromaticity and coupling correction, and effects of machine errors and insertion devices.
\end{abstract}

Presented at the 1999 IEEE Particle Accelerator Conference (PAC99)

New York City, New York, March 29 - April 2, 1999

\footnotetext{
${ }^{1}$ Work supported by Department of Energy contract DE-AC03-76SF00515.
} 


\title{
DYNAMIC APERTURE STUDIES FOR SPEAR $3^{\dagger}$
}

\author{
J. Corbett, $\underline{\text { Y. Nosochkov }}$, J. Safranek, SLAC, Stanford, CA 94309 \\ A. Garren, UCLA, Los Angeles, CA 90095
}

\section{Abstract}

The SSRL is investigating an accelerator upgrade project to replace the present $130 \mathrm{~nm} \cdot \mathrm{rad}$ FODO lattice with an 18 $\mathrm{nm} \cdot \mathrm{rad}$ double bend achromat lattice: SPEAR 3. In this paper, we review the methods used to maximize the SPEAR 3 dynamic aperture including optimization of linear optics, betatron tune, chromaticity and coupling correction, and effects of machine errors and insertion devices.

\section{INTRODUCTION}

SPEAR 3 is a machine upgrade project under study at SSRL [1]. It aims at replacing the current $130 \mathrm{~nm} \cdot \mathrm{rad}$ FODO lattice with an $18 \mathrm{~nm} \cdot \mathrm{rad}$ double bend achromat (DBA) lattice. To reduce the cost of the project and to use the existing synchrotron light beam lines, the new design [2] closely follows the racetrack configuration of the SPEAR tunnel, with the magnet positions fit to the 18 magnet girders. The $3 \mathrm{GeV}$ lattice has two-fold symmetry and periodicity with two identical arcs and two long straight sections.

The lattice functions for one quarter of the ring are shown in Fig. 11. Similar to other light source rings, it has been found advantageous to use combined function bends to relax the optics and reduce sextupole strength.

Though the DBA design has an advantage of a high brightness beam, the strong focusing increases beam sensitivity to machine errors and generates larger chromaticity. The stronger sextupoles increase the amplitude dependent and non-linear chromatic aberrations and reduce the dynamic aperture. It is especially important to maximize the horizontal size of dynamic aperture to minimize the Touschek effect and allow large injection oscillations.

In the following sections we review the lattice optimization and tracking studies. The tracking simulation was done using LEGO [3]. The dynamic aperture was calculated at the symmetry point between arc cells where $\beta_{x} / \beta_{y}=10.1 / 4.8 \mathrm{~m}$.

\section{ERROR FREE LATTICE}

The DBA cell was made to fit the existing $11.7 \mathrm{~m}$ cell length with the magnet positions constrained by the existing photon beam lines and $\sim 3 \mathrm{~m}$ space for the insertion devices (ID). This results in a compact DBA design with stronger focusing and increased sensitivity to machine errors. Vertical focusing added to the bends increased the separation of $x$ and $y$ focusing and reduced strength of the

\footnotetext{
$\dagger$ Work supported by the Department of Energy Contract DE-AC0376 SF00515 and the Office of Basic Energy Sciences, Division of Chemical Sciences.

† E-mail: yuri@slac.stanford.edu.
}

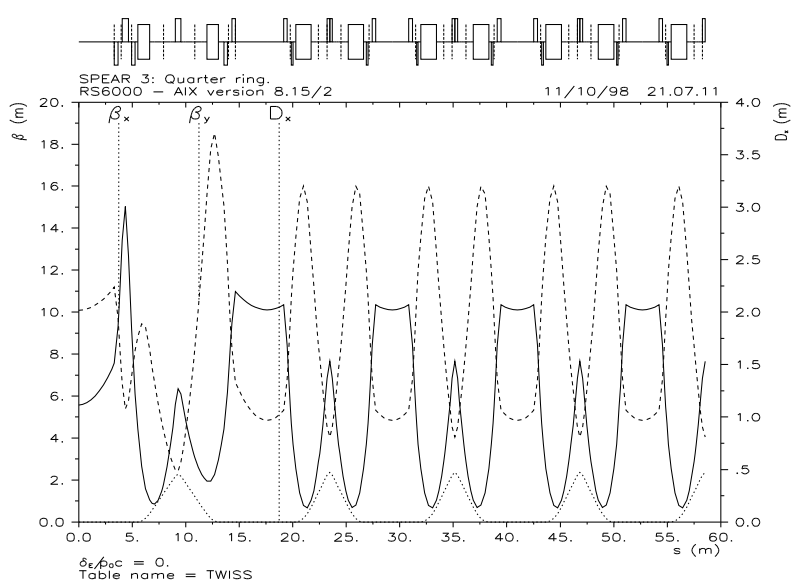

Figure 1: Optics of one quadrant of SPEAR 3.

quadrupoles and sextupoles. Further reduction of the sextupole strengths was achieved by moving the bends as far apart as possible.

The phase advance per arc cell was chosen to be near $\mu_{x}=0.75 \times 2 \pi$ and $\mu_{y}=0.25 \times 2 \pi$. This provides conditions for local cancellation of: 1) geometric aberrations from arc sextupoles located $-I$ apart, and 2) first order chromatic beta waves from sextupoles and quadrupoles located $\pi / 2$ apart, as well as systematic quadrupole errors. With this choice, the total tune would be near $\nu_{x}=14.5$ and $\nu_{y}=5.5$. To move the working tune away from the $1 / 2$ resonance and to minimize resistive wall impedance effects, the tune was moved into the lower quarter on the tune plane $(\nu<1 / 2)$.

The matching cell optics was optimized by performing a horizontal dynamic aperture scan across the matching cell phase advance. The dynamic aperture including $\delta=\frac{\Delta p}{p}$ up to $\pm 3 \%$ was maximized at $\mu_{x, m}=0.78 \times 2 \pi$ and $\mu_{y, m}=$ $0.42 \times 2 \pi$ per matching cell.

To minimize the effect of low order betatron resonances the working tune was chosen slightly below $1 / 4$, away from the 3rd and 4th order resonance lines. The final choice $\left(\nu_{x}=14.19, \nu_{y}=5.23\right)$ was based on favorable horizontal injection conditions and the results of dynamic aperture tune scan. With the chosen tune, the phase advance per arc cell is $\mu_{x}=0.7907 \times 2 \pi$ and $\mu_{y}=0.2536 \times 2 \pi$.

As mentioned previously, the chosen phase advance in the arc cells provides conditions for local compensation of chromatic and geometric aberrations. This scheme would work optimally for the number of arc cells of $4 \times$ integer. With only 7 cells per arc, constrained by the SPEAR geometry, the correction is not complete.

The study showed that chromaticity correction with only 2 sextupole families did not provide adequate dynamic aperture for particles up to $\delta= \pm 3 \%$. Since the 2 families only compensate linear chromaticity, the off-momentum 
Table 1: Systematic rms multipole field errors.

\begin{tabular}{lccc}
\hline Magnet & $\boldsymbol{r}(\boldsymbol{m m})$ & $\boldsymbol{n}$ & $\boldsymbol{\Delta} \boldsymbol{B}_{\boldsymbol{n}} / \boldsymbol{B}$ \\
\hline Dipole & 30 & 2 & $1 \times 10^{-4}$ \\
& & $3-14$ & $5 \times 10^{-4}$ \\
Quadrupole & 32 & $6,10,14$ & $5 \times 10^{-4}$ \\
Sextupole & 32 & 4 & $-8.8 \times 10^{-4}$ \\
& & 5 & $-6.6 \times 10^{-4}$ \\
& & 9 & $-1.6 \times 10^{-3}$ \\
& & 15 & $-4.5 \times 10^{-4}$ \\
\hline
\end{tabular}

aperture is limited by the non-linear chromatic effects. A significant amount of non-linear chromaticity is generated in the matching cells which break periodicity of the 14 arc cells and contribute $\sim 20 \%$ to the total chromaticity. Two additional sextupole families (SFI, SDI) placed in the matching cells reduced the non-linear terms by a factor of 3 and significantly improved the off-momentum aperture.

The matching cell sextupoles also generate geometric aberrations and therefore have to be kept relatively weak in order to preserve the on-momentum aperture. The optimum strengths of the SFI, SDI were evaluated through a horizontal aperture scan versus SFI, SDI strengths.

To increase optical separation between the SF and SD sextupoles two other options were studied. In one option, the SD was combined with part of the adjacent bend. This increased $\beta_{y}$ but reduced dispersion at the SD which led to smaller dynamic aperture. In the second study, the SF was combined with the center quadrupole QFC. This increased dispersion and $\beta_{x}$ at the SF and reduced its strength, but dynamic aperture did not improve.

\section{MACHINE ERRORS}

In tracking simulations, we included random main field errors, random and systematic multipole errors, and random alignment errors. In addition, a skew octupole component was added to skew quadrupoles combined with sextupoles, and a feed-down multipole field was included due to a large trajectory in the bends [\#].

The alignment rms errors for bends, quads and sextupoles used in the study were: $\Delta x, \Delta y=200 \mu \mathrm{m}$, roll $=500 \mu \mathrm{rad}$. The rms main field errors due to differences in magnetic core length were assumed to be (1-2) $10^{-3}$.

The multipole field errors were defined in terms of ratio of the multipole field $\Delta B_{n}$ (normal or skew) to the main magnet field $B$ at radius $r$, where $n=1,2, \ldots$ is the multipole order starting with a bend. The normal systematic and random rms values $\Delta B_{n} / B$ used in the study are listed in Tables 1,2 . Conservatively large values were specified for $n=3,6,10,14$ multipoles on the quads.

The skew quadrupoles physically combined with chromatic sextupoles provided an efficient coupling correction. In total, we used 24 skew quads arranged in 4 families. The induced vertical dispersion was small and far outweighed by the improved aperture. As a future option, the vertical dispersion can be corrected as well by using more independent skew quads. The combined skew quad and sextupole
Table 2: Random rms multipole field errors.

\begin{tabular}{lccc}
\hline Magnet & $\boldsymbol{r}(\boldsymbol{m} \boldsymbol{m})$ & $\boldsymbol{n}$ & $\boldsymbol{\Delta} \boldsymbol{B}_{\boldsymbol{n}} / \boldsymbol{B}$ \\
\hline Dipole & 30 & 2 & $1 \times 10^{-4}$ \\
Quadrupole & 32 & $3,6,10,14$ & $5 \times 10^{-4}$ \\
& & $4,5,7-9,11-13$ & $1 \times 10^{-4}$ \\
Sextupole & 32 & 5 & $1.5 \times 10^{-3}$ \\
& & 7 & $4.8 \times 10^{-4}$ \\
\hline
\end{tabular}

gives rise to a skew octupole field that was systematically added in the simulations.

\section{DYNAMIC APERTURE WITH ERRORS}

For tracking simulations, LEGO first generates a set of magnet errors, then applies tune, orbit, chromaticity and coupling corrections, and finally tracks the particles. For tune correction, two families of doublet quads in the cells were used. Linear chromaticity typically was adjusted to zero with the SF, SD sextupoles. An RF voltage of 3.2 MV was used to generate synchrotron oscillations.

The resultant dynamic aperture without ID's for 6 random seeds of machine errors is shown in Fig. 2. The horizontal dynamic aperture is $18-20 \mathrm{~mm}$ for $\delta= \pm 3 \%$ momentum range. This provides sufficient aperture for a long Touschek lifetime and injection oscillations.

In real machines, the linear chromaticity $\xi=\Delta \nu / \delta$ is typically set slightly positive. Since non-zero chromaticity increases the momentum dependent tune spread, the offmomentum particles would cross more betatron resonances during synchrotron oscillations. Resonance effects can reduce the $\delta \neq 0$ aperture if $\xi$ is large. The $\delta=0$ aperture is affected only by the increased sextupole strength.

Fig. 3 shows dependence of horizontal dynamic aperture on $\xi$ for various $\delta$ (the vertical dependence is similar). In this study, the chromaticity was set equal in both planes. Clearly, for the SPEAR 3 tune the particles lose stability near $1 / 2$ resonance, when $\Delta \nu \approx-0.2$. Though the dynamic aperture for the core beam ( $\operatorname{small} \delta$ ) is not much affected, the Touschek lifetime can be reduced for $\xi>5$.

In the study, typical $\beta$ distortions after correction were $\Delta \beta / \beta< \pm 10 \%$. In a real machine larger modulations can

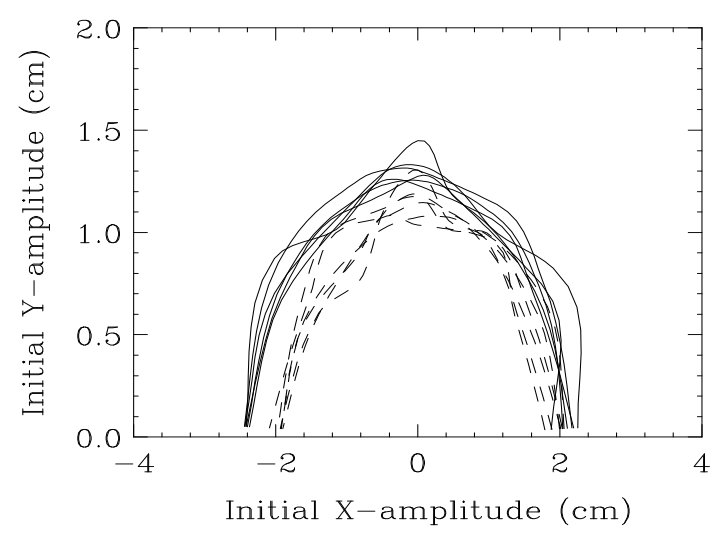

Figure 2: Dynamic aperture for 6 seeds of machine errors for $\delta=0$ (solid) and 3\% (dash). 


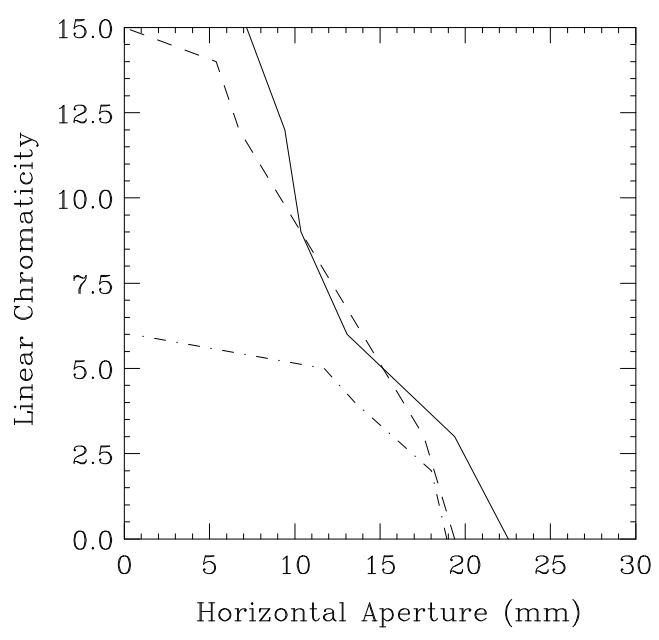

Figure 3: Horizontal dynamic aperture versus linear chromaticity for $\delta=0$ (solid), $1 \%$ (dash) and 3\% (dot-dash).

occur. To verify the effect of large $\beta$ modulation the quad field errors in two matching quad families were increased to generate $\Delta \beta_{x} / \beta_{x} \approx \pm 30 \%$ and $\Delta \beta_{y} / \beta_{y} \approx \pm 20 \%$. The observed aperture reduction was about $15 \%$. Though this aperture is still adequate to operate the machine, such large errors have to be resolved in practice.

The effect of large orbit distortions was studied by including an additional set of uncorrected rms alignment errors. For rms orbit distortions of $\Delta x=3 \mathrm{~mm}, \Delta y=1.5 \mathrm{~mm}$ a maximum of $2 \mathrm{~mm}$ reduction of dynamic aperture was observed for the particles within $\delta< \pm 3 \%$.

Similarly, large sextupole misalignments were studied while the orbit was well corrected. In simulation, $1 \mathrm{~mm}$ rms sextupole misalignments were included which are equivalent to about 10 times the focusing errors from ring quadrupoles. Of the 6 seeds studied, 5 cases showed $>17$ $\mathrm{mm}$ horizontal dynamic aperture for $\delta=0$ particles. The vertical aperture was larger than the $\pm 6 \mathrm{~mm}$ ID chamber size. At $\delta=3 \%$, the horizontal aperture remained $>13 \mathrm{~mm}$.

The actual physical aperture can be a limiting factor for a beam lifetime. In the vertical plane, SPEAR has two ID's with $y= \pm 6 \mathrm{~mm}$ vacuum chamber. In case of strong coupling, the large off-momentum horizontal motion can be transferred into vertical amplitude which could increase beam loss at the vertical physical aperture. To study this effect, we monitored the maximum vertical excursion at an ID location as a function of initial horizontal amplitude. Fig. 4 shows the peak $y$-orbit averaged over 6 random sets of machine errors with $\delta=0-3 \%$ energy oscillations. At 10 $\mathrm{mm}$ injection oscillations, the induced vertical amplitude is below $2 \mathrm{~mm}$ and should not limit the beam lifetime.

In addition to chromatic effects, sextupoles generate geometric aberrations such as amplitude dependent tune shift and high order resonances. Clearly, the dynamic aperture would reach its maximum if the geometric aberrations were zero. One way to verify this limit is to track particles with fixed $\delta=0$ and without sextupoles. The simulation showed that on-momentum aperture could be $40 \%$ larger without sextupole aberrations.

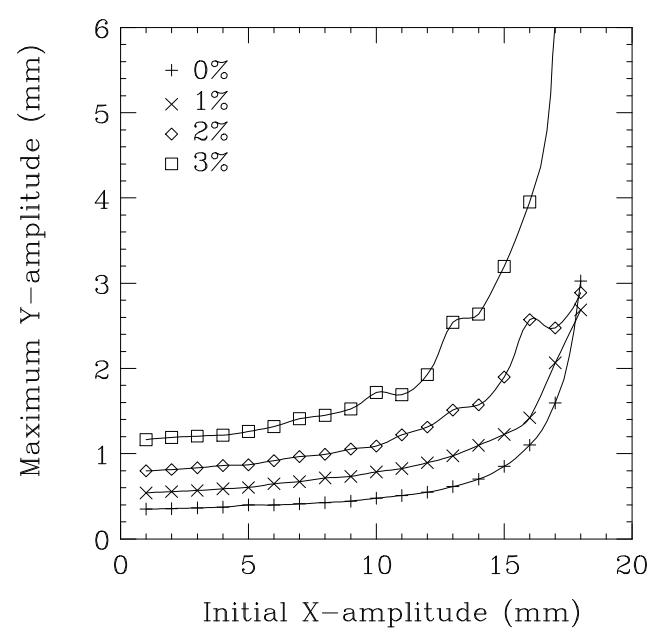

Figure 4: Peak vertical excursion vs initial horizontal amplitude for $\delta=0,1,2,3 \%$.

The geometric effects can be reduced by using 'harmonic' sextupoles placed in non-dispersive regions. Based on analysis in [5] we tested a scheme of two harmonic sextupole families placed in the arc cells. The sextupole strengths were scanned to maximize dynamic aperture. The harmonic correction reduced the amplitude dependent tune shift by about $40 \%$ and the error free dynamic aperture improved by $10-15 \%$. With machine errors, however, the improvement reduces to a minimum. Currently, the harmonic correction is not included in the design.

The fields in insertion devices further reduce the dynamic aperture. The detailed report of wiggler effects in SPEAR 3 is presented in [6]. In summary, the first order wiggler focusing will be locally compensated using cell quadrupoles on either side of a wiggler. Simulations showed that with corrected wigglers, included systematic multipole errors and intrinsic wiggler fields up to dodecapole, the dynamic aperture reduces to about $18 \mathrm{~mm}$ and 8-9 $\mathrm{mm}$ in $x$ and $y$ planes, respectively.

\section{CONCLUSIONS}

Tracking studies combined with optimization of SPEAR 3 lattice show sufficient dynamic aperture for $10 \mathrm{~mm}$ injection oscillations and $>100 \mathrm{hrs}$ of Touschek lifetime. The dynamic aperture results have also been confirmed by $\mathrm{M}$. Borland with the tracking code ELEGANT.

\section{REFERENCES}

[1] R. Hettel, S. Brennan, Synchrotron Radiation News, 11, No. 1 (1998).

[2] SPEAR 3 Conceptual Design Report, in press.

[3] Y. Cai, et al., SLAC-PUB-7642 (1997).

[4] J. Corbett et al., "Multipole Spilldown in the SPEAR 3 Dipole Magnets", these proceedings (1999).

[5] E. A. Crosbie, Proceedings of the 1987 IEEE Part. Acc. Conf., Washington, DC, p. 443 (1987).

[6] J. Corbett, Y. Nosochkov, "Effect of Insertion Devices in SPEAR-3", these proceedings (1999). 